\title{
Lossless Image Compression based on Predictive Coding and Bit Plane Slicing
}

\author{
Haider Al-Mahmood \\ Dept. of Computer Science, \\ Al-Mustansiriya University, \\ College of Science.
}

\author{
Zainab Al-Rubaye \\ Dept. of Computer Science, \\ Baghdad University, \\ College of Science.
}

\begin{abstract}
In this paper, a simple lossless image compression method based on a combination between bit-plane slicing and adaptive predictive coding is adopted for compressing natural and medical images. The idea basically utilized the spatial domain efficiently after discarding the lowest order bits namely, exploiting only the highest order bits in which the most significant bit corresponds to last layer7 used adaptive predictive coding, while the other layers used run length coding. The test results leads to high system performance in which higher compression ratio achieves for lossless system that characterized by guaranty fully reconstruction.
\end{abstract}

\section{General Terms}

Bit-plane slicing along with adaptive predictive coding for lossless image compression.

\section{Keywords}

Image compression, lossless, lossy, predictive coding and bit plane slicing.

\section{INTRODUCTION}

Compression in general represents the enabling technology, lie at the heart of many technologies, such as digital television, DVDs, internet, mobile communications, multimedia, teleconferencing applications, cameras security and other applications. Image compression is a serious issue in storage and transmission because its cuts costs and saves time, basically based on exploits the redundancy in an image such that smaller number of bits can be used to represent the image.

Image compression techniques generally fall into two categories: lossless and lossy. Lossless compression, called Information Preserving or Error Free [1] and also called Noiseless Coding [2], allows an image to be compressed without losing information with low compression ratio that constrained by the image entropy [3]-[5], basically based on the utilization of statistical redundancy alone, thus sometimes referred to as image coding, rather than image compression [6], such as Huffman coding, Arithmetic coding and LempelZiv algorithm. Lossy compression can be considered if wants to have a higher compression ratio while retaining acceptable visual quality for the decompressed image [7], basically based on the utilization of psycho-visual redundancy, either solely or combined with statistical redundancy such as vector quantization, fractal, wavelet and JPEG. Reviews of lossless and lossy techniques can be found in [1,8]-[14]. The utilization of type over the other depends on the application requirements or needs, where lossless suitable for critical applications, like medical image, archiving and satellite imaging whereas lossy more efficient and popular for multimedia applications include image, speech and video.

In order to construct a lossless compression system that guaranty full reconstruction of the compressed image without incurring any distortion (i.e., identical copy) along with high compression ratio, the hybrid system or combined system normally utilized. Predictive Coding (PC) technique, also referred as differential coding is a promising techniques, recently utilized by a number of researchers to compress images due to simplicity, symmetry and efficiency [15]-[20], essentially composed of two consecutive basic steps, start by prediction which means predicting each pixel value from nearby or neighbouring pixels, and then followed by finding the differences between the predicted value and the actual value that called residual or prediction error that is encoded, because of the reduced image information compared to the original image. On the other hand, a useful commonly BitPlane Slicing (BPS) for image compression is adopted [21][24], simply is a separation technique in which the image is sliced at different binary planes or layers according to bit position that efficiently analyzing the relative importance played by each bit of the image [25].

In this paper, an efficient simple and fast hybrid lossless method for compressing images is introduced a combined scheme based on exploited the Bit-Plane Slicing (BPS) for obtains images of various representation corresponding to specific bits, and utilized Predictive Coding (PC) to remove the redundancy between neighbouring pixels, that effectively improve compression ratio. The rest of the paper organized as follows, section 2 explains the proposed system in details; the experimental results and discussion is given in section 3 .

\section{COMBINED LOSSLESS COMPRESSION ALGORITHM}

This section describes the combined or hybrid proposed lossless method to compress an image based on utilizing BitPlane Slicing (BPS) first and then followed by Predictive Coding (PC). The encoder of the suggested system shown in Figure 1 and the implementation is explained in the following steps:

- Step 1: Load the input uncompressed image $I$ of size $N \times N$ that corresponds to grayscale image with 256 colors, 8 bits per pixel.

- Step 2: Perform Bit-Plane Slicing (BPS) to convert the gray scale image into the eight binary images or into eight bit plane images (layers), according to intensity value of each pixel can be represented by an 8-bit binary vector $\left(b_{7}, b_{6}, b_{5}, b_{4}, b_{3}, b_{2}\right.$, $b_{1}, b_{0}$ ), it ranges from Bit level 0 (layer 0 ) which is the Least 
Significant Bit (LSB) to Bit level 7 (layer 7) which is the Most Significant Bit (MSB).

- Step 3: Discard the lower order bits (lowest four) of small or very little contribution of image information without visual degradation of image appearance (i.e., still equivalent to the original image), namely bit planes0-3 (i.e., layer0, layer1, layer2 and layer3) contribute the more subtle details [25]. In other words, use only the higher order bits (top four) of significant major image data of bit planes4-7 (i.e., layer4, layer5, layer6 and layer7), implicitly means reducing the number of bits from 8 bits into 4 bits, that eventually affected in image data compressed in which save more storage space.

- Step 4: Apply Run Length Coding (RLC) to code bit planes46 (i.e., layer4, layer5 and layer6) due to simplicity and efficiency of representation when repeated values presents.

- Step 5: Exploit Predictive Coding (PC) to only most significant bit (MSB) of bit plane7 (layer7) to remove correlation or redundancy embedded between image pixel values. The idea is to predict using neighbour pixels, that works by predicting the pixel value based on the previous values and encoding the differences between the predicted value and the actual value [26]. The simplest form of Predictive Coding (PC) or Differential Coding based on of linear prediction, the predicted value is a neighboring pixel value stored in the predictor. More complicated linear predictors calculate the predicated value as a linear combination of the value of the neighboring pixels [27], various predictors adopted by [28]-[33]. Here the linear combination predictor model utilized, where the following steps are applied:

a) Partition the bit plane7 image $\left(\mathrm{L}_{7}\right)$ into non-overlapping fixed square regions of size $n \times n$ due to simplicity and popularity.

b) Create stationary image $S$ of zero mean block from $\mathrm{L}_{7}$ by computing the mean $m$ of each square fixed block and then subtract the block pixel values from $m$.

$m=\frac{1}{n} \sum_{i=1}^{n} L_{7 i} \ldots \ldots \ldots \ldots \ldots \ldots \ldots \ldots \ldots \ldots \ldots$

$S=L_{7}-m \ldots \ldots \ldots \ldots \ldots(2)$

Where $\mathrm{L}_{7}$ is the layer7 of original image $I, m$ is the block mean of $n$ pixels of block of size $(n \times n)$ (i.e., total number of pixels in fixed square block), and $S$ is the stationary zero mean image.

c) Use an adaptive linear predictor depending on the image features or characteristics, in other words for non-zero mean blocks use predictor of third order model, as shown in Figure 2. Simply, the predictor is a function of neighboring pixels and the coefficients, as in eq. (3). While for zero mean blocks use the simplest form based on previous pixel, which is fast and easy to implement with no need to coefficients estimation.

$\tilde{L}_{7}(x, y)=\left(c_{1} S(x-1, y)+c_{2} S(x, y-1)+c_{3} S(x-1, y-1)\right)$

Where $\tilde{L}_{7}$ is the predicted image layer7, in which each pixel is weighted sum of neighbouring pixels, here represented by top, left and top-left neighbours of each pixel from $S$ stationary image for non-zero mean image block, and $c$ is the predictor coefficients or weights. d) Estimate the predictor coefficients or weights c using the least square method for non zero mean blocks.

$c=\left(P^{T} P\right)^{-1} P^{T} S$

Where $c$ is the coefficient parameters, $p$ is an $(n \times 3)$ matrix of neighbours of each block from $S$ stationary image depending on the prediction model used, $n$ number of rows and 3 represents the number of column applied, where first column corresponding for top neighbour, the second column for left, and third column for top-left neighbours respectively of each pixel of image block.

e) Create the predicted or approximated image value $\tilde{L}_{7}$ using the predictor coefficients or weights, and seed values (overhead information) of neighbour pixels that make use of the first row and column of each block

$\tilde{L}_{7}=P . c$

f) Find the residual or prediction error as difference between the original $\mathrm{L}_{7}$ corresponds to the stationary image $S$ and the predicted one $\tilde{L}_{7}$.

$$
R=S-\tilde{L}_{7}
$$

g) Apply Run Length Coding (RLC) to code the residual image $R$ along with the compressed information that composed of prediction coefficients and seed values.

h) Perform Difference Mean Value (DMV) as the differences between two means values due to correlation embedded between them, as in eq. (7) then followed by Run Length Coding (RLC).

$$
m(i)=m(i)-m(i+1)
$$

Where $i=1,2,3, \ldots, k-1, k$ is size of mean values.

The decoder exploits the coded information that received from the encoder to reconstruct the compressed image as illustrated in Figure 3, starts by reconstructing the mean vales based on adding the values, as reversed to Difference Mean Value (DMV), using the following equation.

$$
m(i-1)=m(i-1)+m(i)
$$

Where $i=k,(k-1),(k-2),(k-3), \ldots, 1$.

Then by utilizing the predictor coefficients or weights and seed values to build or create a predicted image, along with adding the residual image, and finally by adding the other coded bit planes layer (4,5 and 6), so:

$I=\tilde{L}_{7}+R+m+$ CodedBitPleans $(4,5 \& 6)$

Where $I$ is the reconstructed lossless compressed image to be identical to the original image, $\tilde{L}_{7}$ is the predicted image of layer7, $R$ is the residual image of layer7, $\mathrm{m}$ is the mean values, and CodedBitPleans $(4,5 \& 6)$ is the coded information of high order bit planes.

\section{EXPERIMENTAL RESULTS}

Generally, the evaluation of lossless compression system based only on compression performance, normally the compression ratio, which is the ratio of the original image size to the compressed size adopted as the measure of fidelity or the guide of competence of the proposed system, since the decoded compressed image is error free implicitly means identical to the original image. The testing applied on a well- 
known standard images which implies two types of different characteristics or details, the natural type like Lena, Girl, Baboon and Paper, and the medical type of Magnetic Resonance of Brain and Knee, Echo and X-ray of Chest images, (see Figure 4 for an over view), all the images are square of 256 gray levels (8bits/pixel) and of size $256 \times 256$. Figure 4 shows the bit-plane images or layers of tested from least significant bit (LSB) (i.e., layer0) to most significant bit (MSB) (i.e., layer7) of mentioned tested images above.

The results shown in Table 1, using different fixed block sizes of $4 \times 4,8 \times 8$ and $16 \times 16$ of bit- plane7 (layer7) for the tested images. Clearly the results illustrate that the techniques is directly affected by the image's characteristics, since it based on exploiting the spatial domain of the image of both BitPlane Slicing (BPS) and Predictive Coding (PC). In other words, the compression ratio in general varies according to the image nature, where clearly the natural images has much more details than medical images, implicitly meaning a decrease in compression ratio compared to medical one.

The results illustrated that the system efficiency affected by two control parameters, first the high order bit planes used (i.e., layer4, layer5 and layer6) that utilize the run length coding where for low details images of small variation images less byte required, and vice versa. The second is the block size of bit plane7 (i.e., layer7) whereas the block size increase, less coefficient needs (i.e., 3 coefficients for larger block sizes) implicitly improves the compression ratio.

The results have shown that the proposed techniques efficiently works for lossless image compression system type, especially if utilized with multi-resolution techniques and effective encoder that encodes or packs the compressed information in an intelligently way based on combination the statistical based and the dictionary based

\section{ACKNOWLEDGMENTS}

Our thanks to the experts who have contributed towards development of the template.

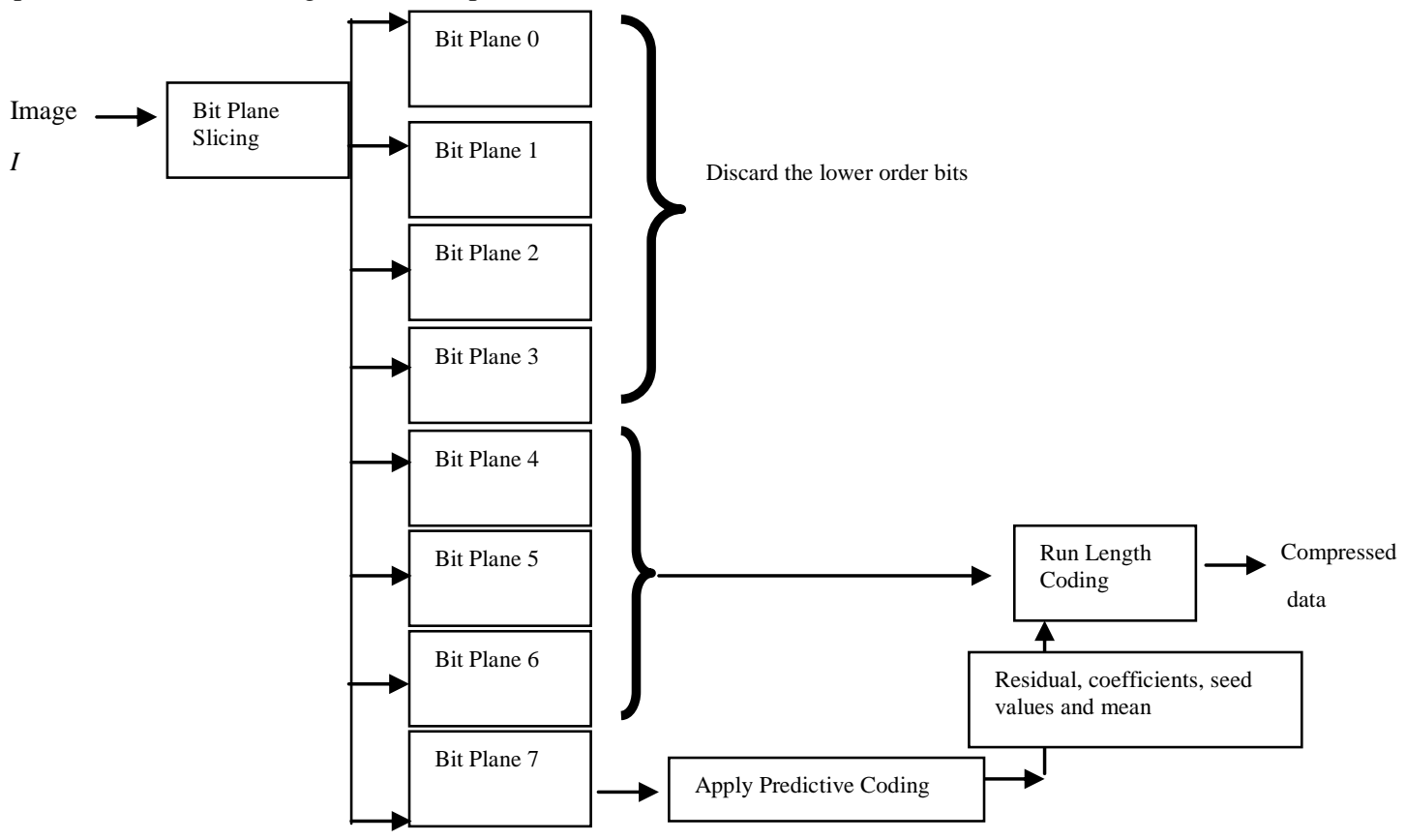

Fig 1: The Encoder (compression) System.

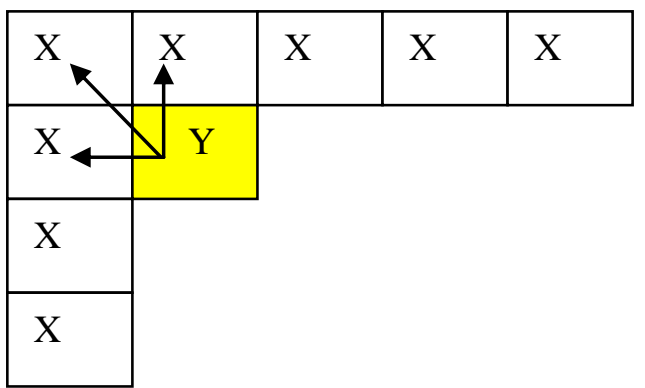

Fig 2: Linear third order predictive model for non-zero mean block, each pixel predicted as a weighted sum of top, left „lefttop neighboring pixels, and the estimated coefficients. 


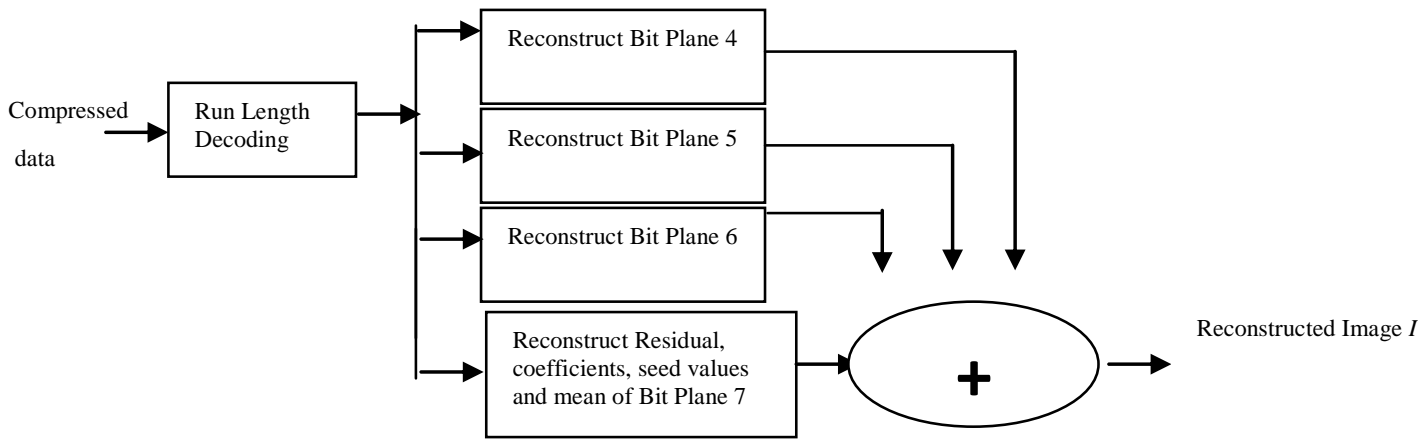

Fig 3: The Decoder (decompression) System.

(a) Natural Images

(b) Medical Images

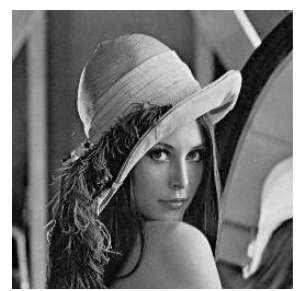

Lena

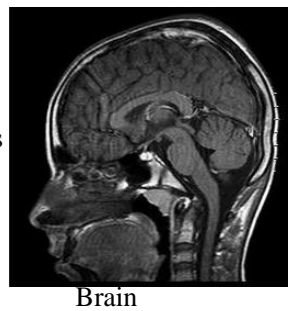

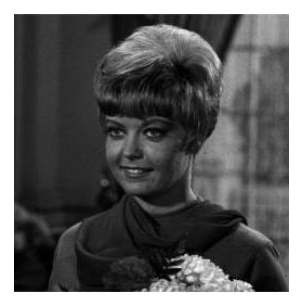

Girl

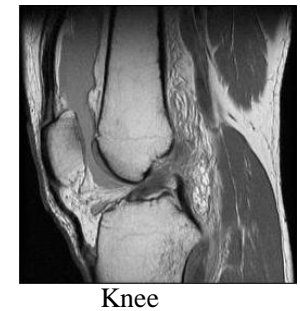

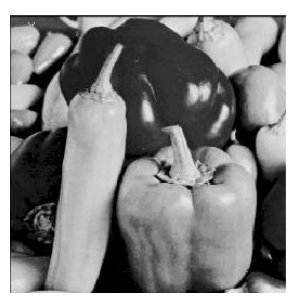

Paper

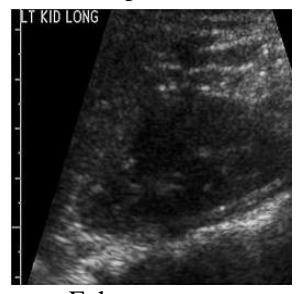

Echo

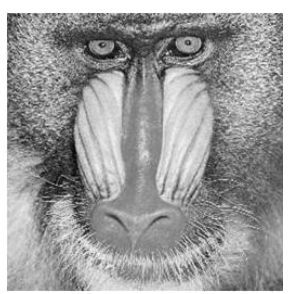

Babbon

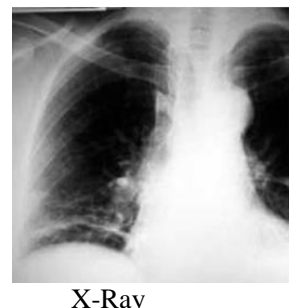

X-Ray

Fig 4: Test images that composed of (a) Natural images of Lena, Girl, Paper and Babbon, and (b) Medical images of Brain, Knee, Echo and C-Ray.

Table 1: Compression ratio of the tested images using different block sizes.

\begin{tabular}{|l|l|l|l|l|l|l|l|}
\hline Tested & Size in bytes of & \multicolumn{2}{|l|}{ Block Size $4 \times 4$} & \multicolumn{2}{l|}{ Block Size $8 \times 8$} & \multicolumn{2}{l|}{ Block Size $16 \times 16$} \\
\cline { 3 - 8 } & Original image & $\begin{array}{l}\text { Size in bytes } \\
\text { compressed } \\
\text { information }\end{array}$ & $\begin{array}{l}\text { Comp. } \\
\text { Ratio }\end{array}$ & $\begin{array}{l}\text { Size in bytes } \\
\text { compressed } \\
\text { information }\end{array}$ & $\begin{array}{l}\text { Comp. } \\
\text { Ratio }\end{array}$ & $\begin{array}{l}\text { Size in bytes } \\
\text { compressed } \\
\text { information }\end{array}$ & $\begin{array}{l}\text { Comp. } \\
\text { Ratio }\end{array}$ \\
\hline Lena & 65536 & 9518 & 6.8855 & 7602 & 8.6209 & 6928 & 9.4596 \\
\hline Girl & 65536 & 5730 & 11.4373 & 4348 & 15.0727 & 3944 & 16.6166 \\
\hline Peppers & 65536 & 7516 & 8.7195 & 5292 & 12.3840 & 4572 & 14.3342 \\
\hline Baboon & 65536 & 12468 & 5.2563 & 9678 & 6.7716 & 8770 & 7.4727 \\
\hline Brain & 65536 & 6924 & 9.4650 & 5588 & 11.7280 & 5196 & 12.6128 \\
\hline Knee & 65536 & 9272 & 7.0682 & 7336 & 8.9335 & 6680 & 9.8108 \\
\hline echo & 65536 & 5870 & 11.1646 & 4536 & 14.4480 & 4142 & 15.8223 \\
\hline x-ray chest & 65536 & 5490 & 11.9373 & 3272 & 20.0293 & 2672 & 24.5269 \\
\hline
\end{tabular}




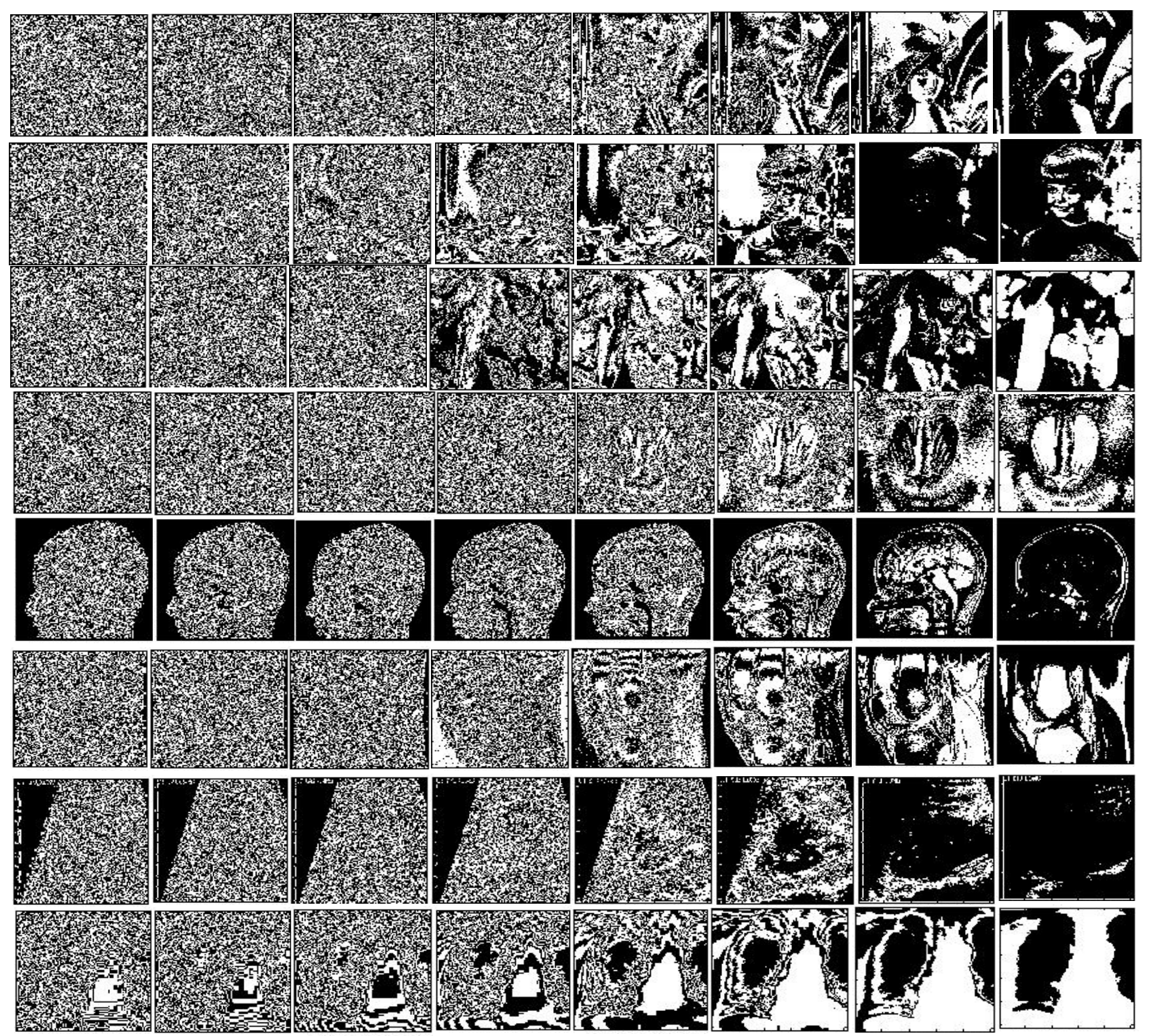

Fig 5: Bit-plane images from layer0 to layer 7 for tested images.

\section{REFERENCES}

[1] Gonzalez, R. C. and Woods, R. E. 2003. Digital Image Processing $2^{\text {nd }}$ edn. Prentice Hall.

[2] Ghalep, N. H. 2004. Image Compression Using Multiresolution Techniques by Wavelet Transform. MSc. thesis, University of AL-Mustansiryah.

[3] Shannon, C. E. 1948. A Mathematical Theory of Communication. Bell System Technology Journal, XXVII(3), 379-423.

[4] Jones, G. A. and Jones, J. M. 2000. Information and Coding Theory. Springer, London.
[5] Baer, M.B. 2006. A General Framework for Codes Involving Redundancy Minimization. IEEE Transactions on Information Theory, 52, 344-349.

[6] Lisa, A. S. 2000. The Mathematical Foundation of Image Compression. MSc. thesis, University of North Carolina, Wilmington.

[7] George, L. E. and Sultan, B. 2011. Image Compression Based on Wavelet, Polynomial and Quadtree. Journal of Applied Computer Science \& Mathematics, 11(5), 15-20.

[8] Nelson, M. and Gailly, J. 1995. The Data Compression Book. $2^{\text {nd }}$ edn. M\&T Books, New York. 
[9] Ghanbari, M. 1999. Video Coding an Introduction to Standard Codecs. Institution of Electrical Engineers, London.

[10] Sayood, K. 2009. Introduction to Data Compression. $3^{\text {rd }}$ edn. Elsevier Publication.

[11] Anitha, S. 2011. 2D Image Compression Technique-A Survey. International Journal of Scientific \& Engineering Research, 2(7), 1-6.

[12] Asolkar, P. S., Zope, P. H. and Suralkar, S. R. 2013. Review of Data Compression and Different Techniques of Data Compression. International Journal of Engineering Research \& Technology (IJERT), 2(1), 1-8.

[13] Amruta, S.G. and Sanjay, L.N. 2013. A Review on Lossy to Lossless Image Coding. International Journal of Computer Applications (IJCA), 67(17), 9-16.

[14] Manjinder, K. and Gaganpreet, K. 2013. A Survey of Lossless and Lossy Image Compression Techniques. International Journal of Advanced Research in Computer Science and Software Engineering, 3(2),323-326.

[15] Das, M. and Burgett, S. 1992. A Lossless Medical Image Compression Scheme Based on Two-Dimensional Space Varying Multiplicative Autoregressive Models. Technical Report TR-92-ESE-06-02, University of Oakland.

[16] Burgett, S. and Das, M. 1993. Predictive Image Coding using Multiresolution Multiplicative Autoregressive Models. Proceedings of the IEEE, 140(2), 127-134.

[17] Das, M. and Lin, C. 1996. Lossless Compression of Medical Images Using Hierarchical Autoregressive Models. $9^{\text {th }}$ IEEE Symposium on Computer-Based Medical Systems, 6-11.

[18] Takamura, S. 1996. Efficient Lossless Still Image Coding Based on the Autoregressive Model. Ph.D. thesis, University of Tokyo.

[19] Chen, Y-T. and Tseng, D-C. 2007. Wavelet-Based Medical Image Compression with Adaptive Prediction. Computerized Medical Imaging and Graphics, 31, 1-8.

[20] Masmoudi, A., Puech, W. and Bouhlel, M. S. 2010. A New Joint Lossless Compression and Encryption Scheme Combining a Binary Arithmetic Coding with a Pseudo Random Bit Generator. International Journal of Computer Science \& Information Security, 8(1), 170175 .

[21] Podlasov, A. and Franti, P. 2006. Lossless Image Compression via Bit-Plane Separation and Multilayer
Context Tree Modeling. Journal of Electronic Imaging, $15,1-1$.

[22] Hisakazu, K., Kunio, F. and Shogo, M. 2009. Simple BitPlane Coding for Lossless Image Compression and Extended Functionalities. PCS'09 Proceedings of the $27^{\text {th }}$ conference on Picture Coding Symposium, 361-364.

[23] Santanu, H., Debotosh, B., Mita, N. and Dipak, K. 2012. A Low Space Bit-Plane Slicing Based Image Storage Method using Extended JPEG Format. International Journal of Emerging Technology and Advanced Engineering, 2(4), 694-699.

[24] Varun, B. and Dalveer, K. 2012. Lossless Compression Technique Using ILZW with DFRLC. International Journal of Computer Science Issues, 9(3), 425-427.

[25] Pasumpon, P. and Sivanandam, N. 2012. Hybrid Algorithm for Lossless Image Compression using Simple Selective Scan order with Bit Plane Slicing. Journal of Computer Science, 8(8), 1338-1345.

[26] Umar, S. 2006. Image Compression using Multiwavelet and DCT Transform. MSc. thesis, Iraqi Commission for Computer and Information Institute for Postgraduate Studies.

[27] Huda, M. 2011. Lossless Image Compression Using Prediction Coding and LZW Scheme. High Diploma thesis, University of Baghdad.

[28] Harrison, C. W. 1952. Experiments with Linear Prediction in Television. Bell System Technology Journal, 31, 764-783

[29] Maragos, P. A., Schafer, R. W. and Mersereau, R. M. 1984. Two-Dimensional Linear Predictive and Its Application to Adaptive Coding of Images. Proceedings of the IEEE international conference on Acoustics, Speech and Signal Processing, 1213-1229.

[30] - Murakami, H., Matsumoto, S., Hatori, Y. and Yamamoto, H. 1987. 15/30Mbit/s Universal Digital TV Codec Using a Median Adaptive Predictive Coding Methods. IEEE Transactions on Communications, 35(6), 637-645.

[31] Salo, J. and Neuvo, Y. 1988. A New Two Dimensional Predictor Design for DPCM Coding of Video Signals. Signal Processing of HDTV, 207-211.

[32] Balram, N. and Moura, J. M. F. 1996. Noncausal Predictive Image Codec. IEEE Transactions on Image Processing, 5(8), 1229-1242. 